\title{
SARS-CoV-2 Rapid Antigen Testing of Symptomatic and Asymptomatic Individuals on the University of Arizona Campus
}

\author{
David T. Harris ${ }^{1,2, *}{ }^{4}$, Michael Badowski ${ }^{1}$, Brandon Jernigan ${ }^{1}$, Ryan Sprissler ${ }^{3}$, Taylor Edwards ${ }^{3}{ }^{\mathbb{D}}$, \\ Randall Cohen ${ }^{4}$, Stephen Paul ${ }^{4}$, Nirav Merchant ${ }^{5}$, Craig C. Weinkauf ${ }^{6}$, Christian Bime ${ }^{7}$, Heidi E. Erickson ${ }^{7}$, \\ Billie Bixby ${ }^{7}$, Sairam Parthasarathy ${ }^{7}$, Sachin Chaudhary ${ }^{7}$, Bhupinder Natt ${ }^{7}$, Elaine Cristan ${ }^{7}$, Tammer El Aini ${ }^{7}$, \\ Franz Rischard ${ }^{7}$, Janet Campion ${ }^{7}$, Madhav Chopra ${ }^{7}$, Michael Insel ${ }^{7}$, Afshin Sam ${ }^{7}$, James L. Knepler ${ }^{7}$, \\ Kenneth Knox ${ }^{8}$, Jarrod Mosier ${ }^{9}{ }^{\circledR}$, Catherine Spier ${ }^{10}$ and Michael D. Dake ${ }^{11}$
}

\section{check for} updates

Citation: Harris, D.T.; Badowski, M.; Jernigan, B.; Sprissler, R.; Edwards, T.; Cohen, R.; Paul, S.; Merchant, N.; Weinkauf, C.C.; Bime, C.; et al. SARS-CoV-2 Rapid Antigen Testing of Symptomatic and Asymptomatic Individuals on the University of Arizona Campus. Biomedicines 2021, 9 , 539. https://doi.org/10.3390/ biomedicines 9050539

Academic Editor: David G. Alleva

Received: 12 April 2021

Accepted: 5 May 2021

Published: 12 May 2021

Publisher's Note: MDPI stays neutral with regard to jurisdictional claims in published maps and institutional affiliations.

Copyright: (c) 2021 by the authors. Licensee MDPI, Basel, Switzerland. This article is an open access article distributed under the terms and conditions of the Creative Commons Attribution (CC BY) license (https:// creativecommons.org/licenses/by/ $4.0 /)$.
1 Biorepository, College of Medicine-Tucson, University of Arizona Health Sciences, University of Arizona, Tucson, AZ 85724, USA; badowski@arizona.edu (M.B.); brandonjernigan@arizona.edu (B.J.)

2 Department of Immunobiology \& Medicine, College of Medicine-Tucson, University of Arizona Health Sciences, University of Arizona, Tucson, AZ 85724, USA

3 College of Medicine-Tucson, University of Arizona Genetics Core, University of Arizona, Tucson, AZ 85724, USA; ryans1@ariziona.edu (R.S.); taylore@arizona.edu (T.E.)

4 Department of Athletic, University of Arizona, Tucson, AZ 85724, USA; rpcohen@arizona.edu (R.C.); spaul@arizona.edu (S.P.)

5 Data Science Institute, University of Arizona, Tucson, AZ 85724, USA; nirav@arizona.edu

6 Department of Surgery, College of Medicine-Tucson, University of Arizona, Tucson, AZ 85724, USA; ccweinkauf@arizona.edu

7 Division of Pulmonary, Allergy, Critical Care and Sleep Medicine, College of Medicine-Tucson, University of Arizona, Tucson, AZ 85724, USA; cbime@arizona.edu (C.B.); herickso@arizona.edu (H.E.E.); bixbyba@arizona.edu (B.B.); sparthasarathy@deptofmed.arizona.edu (S.P.); sachin@deptofmed.arizona.edu (S.C.); bnatt@deptofmed.arizona.edu (B.N.); cristan@deptofmed.arizona.edu (E.C.); telaini@deptofmed.arizona.edu (T.E.A.); rischard@arizona.edu (F.R.); jcampion@deptofmed.arizona.edu (J.C.); chopramv@deptofmed.arizona.edu (M.C.); minsel@deptofmed.arizona.edu (M.I.); asam@deptofmed.arizona.edu (A.S.); jknepler@arizona.edu (J.L.K.)

8 Department of Medicine, University of Arizona-Phoenix, Phoenix, AZ 85724, USA; kknox@arizona.edu

9 Department of Emergency Medicine, College of Medicine-Tucson, Tucson, AZ 85724, USA; jmosier@arizona.edu

10 Department of Pathology, University of Arizona College of Medicine-Tucson, University of Arizona, Tucson, AZ 85724, USA; catherine.spier@bannerhealth.com

11 Office of the Senior Vice-President for Health Sciences, University of Arizona, Tucson, AZ 85724, USA; mddake@arizona.edu

* Correspondence: davidh@arizona.edu; Tel.: +1-(520)-26-5127

Abstract: SARS-CoV-2, the cause of COVID19, has caused a pandemic that has infected more than 80 $\mathrm{M}$ and killed more than 1.6 M persons worldwide. In the US as of December 2020, it has infected more than $32 \mathrm{M}$ people while causing more than 570,000 deaths. As the pandemic persists, there has been a public demand to reopen schools and university campuses. To consider these demands, it is necessary to rapidly identify those individuals infected with the virus and isolate them so that disease transmission can be stopped. In the present study, we examined the sensitivity of the Quidel Rapid Antigen test for use in screening both symptomatic and asymptomatic individuals at the University of Arizona from June to August 2020. A total of 885 symptomatic and 1551 asymptomatic subjects were assessed by antigen testing and real-time PCR testing. The sensitivity of the test for both symptomatic and asymptomatic persons was between 82 and $90 \%$, with some caveats.

Keywords: rapid antigen test; PCR; SARS-CoV-2; asymptomatic; diagnostic screening 


\section{Introduction}

In less than one year, the novel coronavirus, SARS-CoV-2, the causative agent of COVID-19, has jumped from animals to humans, causing a pandemic that has infected more than $80 \mathrm{M}$ and killed more than $12 \mathrm{M}$ persons worldwide. In the US alone, as of February 2021, it has infected more than $32 \mathrm{M}$ people while causing approximately 570,000 deaths [1]. Both sets of numbers continue to mount daily, with fears of a surge of infections ever-present. As the pandemic persists, there has been great public demand to "reopen" cities, workspaces and places of social interaction such as schools and university campuses despite the risks involved. To consider these demands it is necessary to rapidly identify the virus-infected individuals and isolate them so that disease transmission can be stopped.

Tracking and tracing efforts that are required to establish the identity of secondary infections depend on the rapid identification of the primary infected person to limit viral spread. The source of infection must be isolated and quarantined so they may be observed and treated as necessary. Viral testing strategies must be specific for the disease, sensitive to low (possibly asymptomatic) viral loads and return results to those responsible in as short a time as possible [2]. Typically, polymerase chain reaction (PCR) assays have been the gold standard for viral diagnostic testing [3,4], but other testing options are available. Unfortunately, the PCR test is not generally a point-of-care test and often takes $48-72 \mathrm{~h}$ to return results, is relatively expensive at scale, has been plagued by reagent scarcity and may even be too sensitive at times [2-4].

There are other testing options, however, that may be sufficiently sensitive and diseasespecific to achieve the rapid identification of infected patients and facilitate early isolation and contact tracing. Such tests may be able to compensate for reduced sensitivity as compared to PCR testing by being amenable to multiple rounds of testing combined with the rapid return of results within minutes to hours. As long as these alternative testing approaches are safe, easy to perform and inexpensive, there could be significant advantages. If these tests are capable of being self-administered and include the return of results documentation, then such tests might become a preferred choice when screening large populations of individuals in a high throughput manner.

Such a testing strategy became commercially available from Quidel, Inc. (San Diego, CA, USA) to analyze subjects for the presence of the SARS-CoV-2 viral antigen Ag) [5]. The assay (Sofia SARS Antigen FIA) detects the presence of antigen produced by a live virus. The test reports the result within 15-30 min, can be scaled to perform thousands of tests per day, has US Food and Drug Administration (FDA) Emergency Use Authorization (EUA) for symptomatic subjects, is relatively inexpensive at USD $23 /$ test, and can be performed using self-administered (anterior) nasal swabs. Quidel is a company with a history of producing FDA-approved rapid diagnostic tests (such as for influenza), lending confidence to purported claims. The testing device, the Sofia 2, has an internal storage device, along with a flash/USB reporting option, and data can be directly exported to the cloud via $3 \mathrm{G}$ connections for inclusion in subject medical records. Quidel has reported the sensitivity of its SARS-CoV-2 rapid Ag test to be $93 \%$ with near $100 \%$ specificity [5], (FDA EUA Application) but these results were not based on testing large numbers of "real-world" samples.

In the present study, we examined the sensitivity of the Quidel Rapid Ag test for use in screening both symptomatic and asymptomatic individuals in the student and staff community at the University of Arizona from June to August 2020. After test validation, a total of 885 symptomatic and 1551 asymptomatic subjects were assessed by both antigen testing and real-time PCR (RT-PCR or PCR) testing performed side-by-side at the same time on the same patient.

\section{Materials and Methods}

\subsection{Subjects}

All human subjects in the study were students, faculty, or staff at the University of Arizona. Participation was voluntary. Symptomatic subjects were tested within 5 days of 
symptom onset. Asymptomatic subjects were asked to volunteer for testing as a screen for return to campus activities, and none had symptoms of infection or had been exposed to anyone with symptoms. All testing was performed by CLIA and/or CAP-accredited laboratories under the standard of care. An institutional review determined that the study did not require consent as long as all data was de-identified upon receipt and that all data pertaining to this study were de-identified prior to publication. All collections were performed between June and August 2020. In addition, a small number of COVID-19positive patients from the local university ICU provided samples as part of the initial validation studies of the assay (with consent).

\subsection{Biospecimen Collection}

Rapid antigen (Ag) testing specimens were collected using foam swabs provided by the manufacturer. Dry anterior nasal collections were self-performed for both nostrils under supervision. Dry swabs were placed in a barcoded $15 \mathrm{~mL}$ conical tube, kept in an insulated cooler to maintain $65-80^{\circ} \mathrm{F}\left(18-27^{\circ} \mathrm{C}\right)$ and transported within $60 \mathrm{~min}$ of collection to the laboratory. PCR specimens were collected from one nostril using CDC-approved nasopharyngeal (NP) swabs (Miraclean \#MRC 96000, Tongle, Longgang, Shenzhen, China) with medical assistance. The swab was then placed in a VMT transport buffer and brought to the laboratory for testing within $60 \mathrm{~min}$. NP and Ag nasal specimens were collected in that order at the same time from individuals in this study. Tested subjects were classified as being antigen-positive/PCR-positive, antigen-negative/PCRnegative, antigen-positive/PCR-negative or antigen-negative/PCR-positive based on the combination of test results.

\subsection{PCR Testing}

PCR testing utilized a CDC-approved primer set with a detected/not detected cut-off of 40 cycles (CDC 2019-Novel Coronavirus (2019-nCoV) Real-Time RT-PCR Diagnostic Panel (N1, N2 and RP primer set) ordered through Integrated DNA Technologies, Inc. (IDT): Catalog \#2019-nCoVEUA-01 Diagnostic Panel Box \#1; (https: / / www.cdc.gov / coronavirus / 2019-ncov / lab/virus-requests.html) (accessed on 1 February 2021) [6,7]. Tests were performed according to the manufacturer's instructions.

\subsection{Rapid Antigen Testing}

The Quidel Sofia 2 SARS antigen FIA test displayed a digital readout as positive or negative based on the ratio of relative fluorescence units to background in an LFT (lateral flow test) assay termed the S/CO (signal versus control) ratio [5]. Ratios above 1.0 are considered positive results, and ratios can range from 1-600 depending on the level of viral infection (viral load). Samples were processed according to the manufacturer's instruction within 90 min of arrival at the laboratory and analyzed using the Sofia 2 instrument.

\section{Results}

The sensitivity and specificity of the rapid Ag assay were initially assessed by performing rapid antigen testing using three types of biospecimens: "spiked" positive and negative samples provided by Quidel, Inc.; samples obtained from suspected, symptomatic, critically ill patients admitted to intensive care units; and samples obtained from asymptomatic campus students and staff (Table 1). Anterior nasal samples were collected with dry swabs, processed and tested as per the manufacturer's instructions. PCR testing specimens were simultaneously collected using NP swabs, placed into a viral transport media (VTM), processed and analyzed within $12 \mathrm{~h}$. Concordant results were observed for rapid antigen testing and PCR testing for the majority of samples examined (32 of 35 samples), whether spiked samples, suspected COVID-19-positive samples or asymptomatic patients were analyzed. Discordant findings where subjects were antigen-negative but PCR-positive for the presence of the virus were observed for specimens with high PCR cycle thresholds (of 31 and above). The overall results obtained in these studies provided a determination 
of $91.4 \%$ sensitivity with $100 \%$ specificity for the antigen test, similar to that claimed by the vendor.

Table 1. Validation of the SARS-CoV-2 rapid antigen test.

\begin{tabular}{|c|c|c|c|}
\hline ID\# & Type of Sample & Expected Result & Ag Test Result \\
\hline 1 & SPK & POS & POS \\
\hline 2 & SPK & NEG & NEG \\
\hline 3 & SPK & POS & POS \\
\hline 4 & SPK & POS & POS \\
\hline 5 & SPK & POS & POS \\
\hline 6 & SPK & POS & POS \\
\hline 7 & SPK & NEG & NEG \\
\hline 8 & SPK & POS & POS \\
\hline 9 & SPK & POS & POS \\
\hline 10 & SPK & POS & POS \\
\hline 11 & SPK & POS & POS \\
\hline 12 & SPK & NEG & NEG \\
\hline 13 & SPK & NEG & NEG \\
\hline 14 & SPK & POS & POS \\
\hline 15 & SPK & POS & POS \\
\hline 16 & SPK & POS & POS \\
\hline 17 & SPK & POS & POS \\
\hline 18 & SPK & NEG & NEG \\
\hline 19 & SPK & POS & POS \\
\hline 20 & SPK & POS & POS \\
\hline ID\# & Type of Sample & Ag Test & RT-PCR (ct) \\
\hline BF00043 & SYMP & POS & POS (ct 23) \\
\hline BF00044 & SYMP & POS & POS (ct 14) \\
\hline BF00081 & SYMP & POS & POS (na) \\
\hline BF00094 & SYMP & POS & POS (na) \\
\hline BF00106 & SYMP & POS & POS (na) \\
\hline BF00112 & SYMP & POS & POS (na) \\
\hline 22 & ASYM & POS & POS (ct 27) \\
\hline 34 & ASYM & POS & POS (ct 22) \\
\hline 113 & ASYM & POS & POS (ct 25) \\
\hline 127 & ASYM & POS & POS (ct 17) \\
\hline 156 & ASYM & POS & POS (ct 22) \\
\hline 112 & ASYM & NEG & POS (ct 36) \\
\hline 139 & ASYM & NEG & POS (ct 31) \\
\hline 24524 & ASYM & POS & POS (ct 23) \\
\hline$\underline{21448}$ & ASYM & NEG & POS (ct 35) \\
\hline
\end{tabular}

A mixed set of possible positive and negative nasal swabs was analyzed as part of the validation of the rapid antigen test. Nasal swabs were collected as per the manufacturer's instructions from "spiked" samples (SPK), symptomatic critically ill patients hospitalized in an intensive care unit (SYMP), and asymptomatic (ASYM) subjects from the campus community. Additional subjects that tested negative by both Ag and PCR tests during the validation studies are not shown. ct: PCR cycle threshold. POS: positive result/detected. NEG: negative result/not detected. ID\#: unique sample identification number. na: ct not available due to HIPAA restrictions. Discordant results are shown in bold type.

A select group of initially asymptomatic patients from the validation studies who tested positive during the validation studies were followed and tested for an additional 1-2 weeks using the rapid antigen test and PCR test as before. As shown in Table 2, during subsequent testing once PCR cycle thresholds (ct) for locus N1 amplification were higher than 31 cycles, the rapid antigen test was unable to detect the presence of the virus. Preliminary results from such subjects have indicated that anti-virus antibody titers were present during those later test times when subjects displayed high ct values and that patients may not be contagious due to low viral loads or the absence of live virus. Although preliminary, these results may also suggest that the shedding of live virus as indicated by a 
positive rapid antigen test may only persist for a week (or so), despite continued reactivity by viral PCR analysis.

Table 2. Longitudinal study of selected asymptomatic subjects.

\begin{tabular}{ccc}
\hline ID\# & Ag Test & RT-PCR (Cycle Threshold) \\
\hline 22 & POS & POS (ct27) \\
& NEG & POS (ct33) \\
& NEG & POS (ct34) \\
\hline 113 & POS & POS (ct25) \\
& NEG & POS (ct35) \\
\hline 127 & POS & POS (ct17) \\
& NEG & POS (ct36) \\
& NEG & POS (ct35) \\
\hline 112 & NEG & POS (ct36) \\
& NEG & POS (ct36) \\
\hline 139 & NEG & POS (ct31) \\
& NEG & POS (ct36)
\end{tabular}

During the validation studies, several subjects were tested weekly for the virus over a period of 2-3 consecutive weeks, simultaneously by both antigen and PCR testing, with results shown above (tests conducted 1 week apart). At this time there were also additional samples that tested negative by both antigen and PCR tests that are not shown for the sake of clarity.

To explore the limits of the rapid antigen assay, 885 students and staff presenting at university campus health were tested, including both those exposed to the virus (i.e., in close contact with someone that tested positive for the virus) and those symptomatic with cough, fever, aches, etc. were tested. As shown in Table 3, more than half of all subjects $(64.8 \%)$ presenting with symptomatic complaints were negative for the virus by both the PCR and rapid antigen tests. A total of 305 individuals tested positive by PCR (34.5\%) while a total of 258 persons tested positive by the rapid antigen test $(29.2 \%)$. Discordant PCR and rapid antigen testing results were once again observed in biospecimens with high PCR cycle thresholds (average ct of 34). It appeared that the Quidel rapid antigen test did not detect approximately $6 \%$ of the positive results detected by the PCR test. These samples displayed cts with 8 cycles higher on average. This finding implies that the COVID-19-positive samples that the Quidel test "missed" contained on average 256 times less viral load than the positive samples it could detect. Calculations from these results indicated a sensitivity of $82.3 \%$, while the specificity of the rapid antigen assay was $98.2 \%$ based on a finding of 7 antigen-positive, PCR-negative results out of 885 total results. The positive predictive value (PPV) and negative predictive value (NPV) were calculated to be $97.3 \%$ and $91.4 \%$, respectively. A similar assessment to that described was performed with a large sampling of the university campus staff and student body as shown in Table 3 . All subjects were randomly selected and asymptomatic at the time of both PCR and rapid antigen testing. The vast majority of subjects were virus-negative by both the PCR and the rapid antigen test (98\%), as might be expected. 
Table 3. Relationship between PCR cycle threshold and rapid antigen test reactivity.

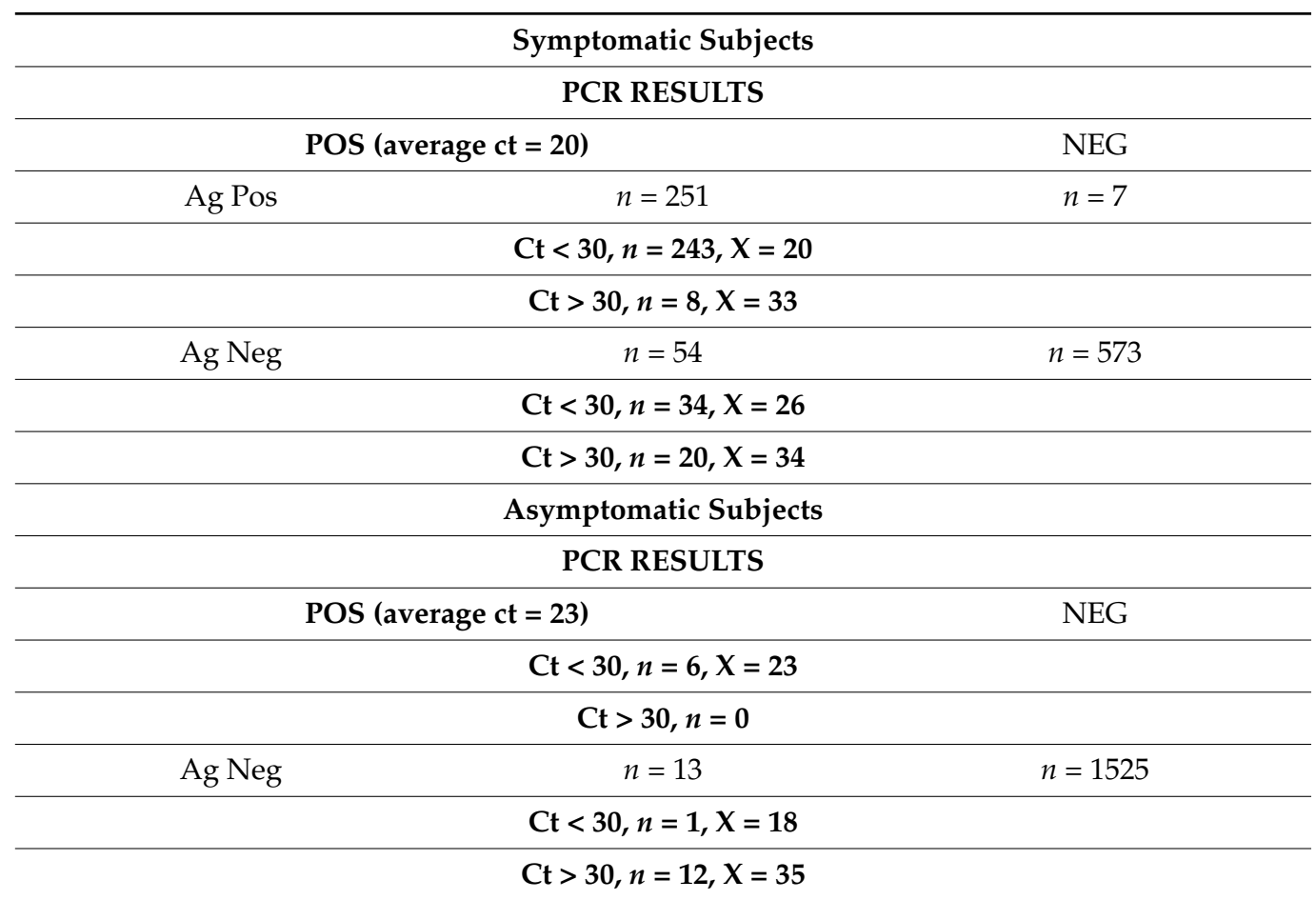

Data presented in Table 3 are stratified according to PCR cycle thresholds (ct). Subjects that tested PCR-positive and Ag-negative (in bold) were divided into two groups: $<30 \mathrm{ct}$ and $>30 \mathrm{ct}$ and mean $(X) \mathrm{ct}$ values were calculated.

Based on these findings, further analyses were performed to examine the role of PCR cycle time (i.e., potential viral load) in the detection of the virus by the rapid antigen test. It was observed that symptomatic patients displayed lower cycle thresholds in the PCR analysis (see Figure 1) regardless of whether they were also Ag-positive for the virus. Reasons for this observation are not straightforward, but it may be that some SARS-CoV-2 infections are missed due to sampling or that lower PCR cycle thresholds may be a result of an unrecognized inflammatory immune reaction. Regardless, symptomatic patients seemed to require fewer PCR cycle times to detect the virus. Violin dot plots of the findings (Figure 1) demonstrated that subjects that were PCR-positive but Ag-negative displayed cycle times clustering at the higher end of the scale.

The results observed in Figure 1 led us to further examine the sensitivity of the rapid Ag test in asymptomatic subjects. Table 3 dissects the effects of PCR cycle threshold on test concordance in more detail. In each of the 4 groups, the subjects with ct $>30$ were segregated from the other subjects in each of the populations. As shown, this stratification resulted in 243 out of $277(88 \%)$ symptomatic subjects and 6 out of $7(86 \%)$ asymptomatic subjects with cycle times below 30 being both Ag and PCR test concordant. These analyses confirmed the conclusions implied in Figure 1 demonstrating that the rapid antigen test was insensitive to samples with a ct higher than 30 . The calculated specificity of the assay for asymptomatic patients remained at $99 \%$. 


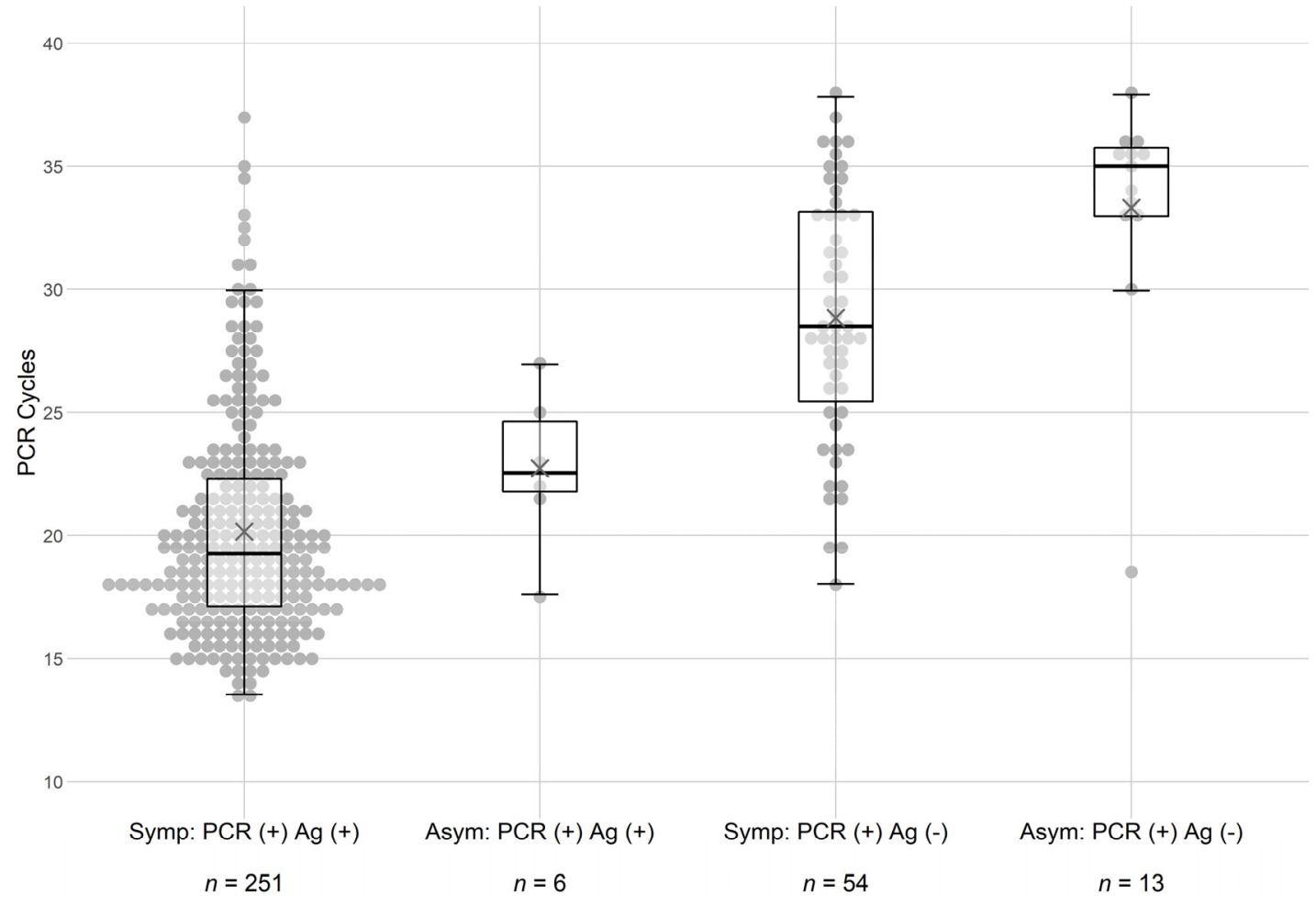

Figure 1. Comparison of PCR cycle thresholds in symptomatic and asymptomatic patients. Subjects were tested simultaneously by Ag and PCR testing for SARS-CoV-2. Patients were broadly grouped into symptomatic patients presenting at campus health and asymptomatic subjects on the university campus. Subgroup results were analyzed based on the combination of results for each test. Violin plots of the data are shown. Each circle represents an independent measurement for a subject.

\section{Discussion}

Overall results from the rapid antigen and PCR tests were generally concordant for these two large groups of individuals on the university campus, regardless of symptomology, when PCR ct were 30 cycles or less. Of note, asymptomatic subjects had higher underlying PCR cycle thresholds regardless of whether they were antigen-positive or negative, which has also been reported in a previous study [8]. Possibly the most significant observation from the study was that subjects missed with the rapid Ag test were generally only those with a PCR cycle threshold higher than 30 cycles, and possibly not infectious and/or less sick [9]. A ct of 30 cycles was chosen as the cut-off threshold as it reflects a potential viral load of 1000 virus particles, the minimum level thought to be needed for infectivity [10]. Overall, our findings based on validation samples as well as the analyses of symptomatic and asymptomatic samples indicated a sensitivity between 86 and $92 \%$ and a specificity of $97-99 \%$ for the rapid antigen test, for those individuals that are both infected and contagious with cycle thresholds under 30 . That is, in symptomatic individuals the $\mathrm{Ag}$ test is very sensitive, while in asymptomatic individuals the Ag test detected less than half of those who had been infected with SARS-CoV-2 but identified $86 \%$ of those who were contagious and likely to spread the virus. In addition, based on the findings, a slightly higher false-negative rate for asymptomatic persons with the rapid antigen test could be overcome if one were to perform antigen testing a minimum of three times a week. Repeated testing could reduce to less than one percent the chance that any individual who is truly positive is missed by the antigen test [11].

Interestingly, more than half of all subjects who presented at Campus Health with COVID-19 symptoms during the study period did not test positive for SARS-CoV-2 by either a rapid antigen or PCR test, a noteworthy and cautionary finding in and of itself. This finding also helps to explain the lower-than-expected positivity rates in the symptomatic 
population. Our observations seem to indicate that compared with the rapid antigen test the PCR test detects viral loads that may be 200 -fold lower, which may not be biologically relevant in terms of infectivity/contagiousness $[9,10]$. Recent evidence suggests that a PCR ct of 30 is the threshold for SARS-CoV-2 infectivity/contagiousness [10,12,13]. In fact, the rapid Ag test and the PCR test were generally concordant in their findings, although PCR testing may be overly sensitive (and the cut-off for PCR positivity may need to be lower than 40 cycles). The rapid Ag test may be best for determining if subjects are contagious and most likely to spread the virus, while the PCR test may be a better indicator for general infection and prevalence of the virus in the general population. Overall, the rapid antigen test seems to be sufficient and more than adequate for rapid screening of both asymptomatic and symptomatic subjects. Importantly, the rapid antigen test can easily be scaled up to test 2000-3000 subjects per day without a considerable increase in staff and equipment, making it amenable to high-throughput, large scale screening efforts (based on our own experience at the University of Arizona where we have now administered and performed more than 200,000 such rapid Ag tests). It also can be readily used for the daily screening of select populations with only a small decrease in sensitivity as compared to PCR testing. The decreased sensitivity can be compensated for with the increased test frequency of those individuals. However, it must be remembered that both the rapid Ag test and the PCR test are point-in-time tests, with no guarantee that one does not become infected moments to hours to days after sample collection, emphasizing the need for face coverings and social distancing to finally stem the pandemic until a vaccine becomes widely available.

Author Contributions: Conceptualization: D.T.H. and M.B. Methodology: B.J., R.S., T.E., R.C. and S.P (Stephen Paul) Software: N.M. Validation: D.T.H., M.B., B.J., R.S. and T.E. Formal analysis: C.C.W., C.B., H.E.E., B.B., S.P (Stephen Paul), S.C., B.N., E.C., T.E.A., F.R., J.C., M.C., M.I., A.S., J.L.K., K.K., J.M., C.S. and M.D.D. Writing: D.T.H. Review and editing: C.C.W., C.B., H.E.E., B.B., S.P. (Sairam Parthasarathy), S.C., B.N., E.C., T.E.A., F.R., J.C., M.C., M.I., A.S., J.L.K., K.K., J.M., C.S. and M.D.D. All authors have read and agreed to the published version of the manuscript.

Funding: This research received no external funding.

Institutional Review Board Statement: Ethical review and approval were waived for this study, due to testing being performed under the standard of care using EUA-approved diagnostic tests.

Informed Consent Statement: Not applicable.

Data Availability Statement: Not applicable.

Acknowledgments: We wish to acknowledge the invaluable participation of David Salafsky and Michael Stilson from the University of Arizona Campus Health, without whom it would not have been possible to conduct these studies. In addition, we acknowledge all the staff involved with biospecimen collections as well as the technicians who toiled many hours to analyze the samples once they arrived in the laboratory. Finally, we thank all of the subjects for their participation.

Conflicts of Interest: The authors declare no conflict of interest.
Abbreviations
CAP College of American Pathology
CDC Center for Disease Control
CLIA Clinical Laboratory Improvement Amendments
ICU Intensive care unit

\section{References}

1. WHO Coronavirus (COVID-19) Dashboard. Available online: www.COVID19.who.int (accessed on 1 February 2021).

2. Wee, S.K.; Sivalingam, S.P.; Yap, E.P.H. Rapid Direct Nucleic Acid Amplification Test without RNA Extraction for SARS-CoV-2 Using a Portable PCR Thermocycler. Genes 2020, 11, 664. [CrossRef] [PubMed]

3. Mackay, I.M. Real-time PCR in virology. Nucleic Acids Res. 2002, 30, 1292-1305. [CrossRef] [PubMed] 
4. Hernández-Huerta, M.T.; Mayoral, L.P.; Navarro, L.M.S.; Mayoral-Andrade, G.; Mayoral, E.P.; Zenteno, E.; Pérez-Campos, E. Should RT-PCR be considered a gold standard in the diagnosis of COVID-19? J. Med. Virol. 2021, 93, 137-138. [CrossRef] [PubMed]

5. HCP Fact Sheet. Available online: www.fda.gov/media/137884/download (accessed on 1 February 2021).

6. Research Use Only 2019-Novel Coronavirus (2019-nCoV) Real-Time RT-PCR Primers and Probes. Available online: www.cdc. gov/coronavirus / 2019-ncov/lab/rt-pcr-panel-primer-probes.html (accessed on 1 February 2021).

7. CDC 2019-Novel Coronavirus (2019-nCoV) Real-Time RT-PCR Diagnostic Panel. Available online: www.fda.gov/media/134922 / download (accessed on 1 February 2021).

8. Kociolek, L.K.; Muller, W.J.; Yee, R.; Bard, J.D.; Brown, C.A.; Revell, P.A.; Wardell, H.; Savage, T.J.; Jung, S.; Dominguez, S.; et al. Comparison of Upper Respiratory Viral Load Distributions in Asymptomatic and Symptomatic Children Diagnosed with SARS-CoV-2 Infection in Pediatric Hospital Testing Programs. J. Clin. Microbiol. 2020, 59. [CrossRef] [PubMed]

9. Mina, M.J.; Parker, R.; Larremore, D.B. Rethinking Covid-19 Test Sensitivity-A Strategy for Containment. N. Engl. J. Med. 2020, 383, e120. [CrossRef] [PubMed]

10. Liu, W.-D.; Chang, S.-Y.; Wang, J.-T.; Tsai, M.-J.; Hung, C.-C.; Hsu, C.-L.; Chang, S.-C. Prolonged virus shedding even after seroconversion in a patient with COVID-19. J. Infect. 2020, 81, 318-356. [CrossRef] [PubMed]

11. Larremore, D.B.; Wilder, B.; Lester, E.; Shehata, S.; Burke, J.M.; Hay, H.A.; Tambe, M.; Mina, M.H.; Parker, R. Test sensitivity is secondary to frequency and turnaround time for COVID-19 surveillance. medRxiv 2020. [CrossRef]

12. SARS-CoV-2 Infectious Dose. Available online: http://www.clinlabnavigator.com/sars-cov-2-infectious-dose.html (accessed on 1 February 2021).

13. Pekosz, A.; Cooper, C.K.; Parvu, V.; Li, M.; Andrews, J.C.; Manabe, Y.; Kodsi, S.; Leitch, J.; Gary, D.S.; Roger-Dalbert, C. Antigen-based testing but not real time PCR correlates with SARS-CoV-2 virus culture. medRxiv 2020. [CrossRef] 